\title{
La zone grise, le Gros mot, et de nouvelles manières de le raconter : quelques histoires récentes de la Grande Famine
}

The grey zone, the G-word, and new ways of telling it: Some recent histories of the Great Famine

\section{Breandán Mac Suibhne}

Traducteur : Chloé Lacoste

\section{CpenEdition} Journals

Édition électronique

URL : http://journals.openedition.org/rfcb/282

DOI : $10.4000 / \mathrm{rfcb} .282$

ISSN : 2429-4373

Éditeur

CRECIB - Centre de recherche et d'études en civilisation britannique

\section{Édition imprimée}

Date de publication : 1 septembre 2014

Pagination : 213-238

ISSN : 0248-9015

\section{Référence électronique}

Breandán Mac Suibhne, «La zone grise, le Gros mot, et de nouvelles manières de le raconter: quelques histoires récentes de la Grande Famine », Revue Française de Civilisation Britannique [En ligne], XIX-2 | 2014, mis en ligne le 01 mai 2015, consulté le 05 mai 2019. URL : http:// journals.openedition.org/rfcb/282 ; DOI : 10.4000/rfcb.282

Ce document a été généré automatiquement le 5 mai 2019.

\section{c) (†) $\odot$}

Revue française de civilisation britannique est mis à disposition selon les termes de la licence Creative Commons Attribution - Pas d'Utilisation Commerciale - Pas de Modification 4.0 International. 


\title{
La zone grise, le Gros mot, et de nouvelles manières de le raconter : quelques histoires récentes de la Grande Famine
}

\author{
The grey zone, the G-word, and new ways of telling it: Some recent histories of \\ the Great Famine
}

Breandán Mac Suibhne

Traduction : Chloé Lacoste

\section{NOTE DE L'ÉDITEUR}

Une version plus longue de l'article est disponible en anglais sur le site de la Dublin Review of Books : <http://www.drb.ie/ESSAYS/a-jig-in-the-poorhouse>.

\section{NOTE DE L'AUTEUR}

Le « Gros mot » traduit ici l'anglais G-word, qui désigne le mot tabou : le génocide.

Les ouvrages abordés ici sont les suivants : Tim Pat Coogan, The Famine Plot : England's role in Ireland's Greatest Tragedy, Houndmills et New York : Palgrave Macmillan, 2012 ; Enda Delaney, The Curse of Reason : The Great Irish Famine, Dublin : Gill and Macmillan, 2012 ; John Kelly, The Graves are Walking: The Great Famine and the Saga of the Irish People, Londres : Faber and Faber, 2012 / New York : Henry Holt, 2012 ; Ciarán Ó Murchadha, The Great Famine: Ireland's Agony, Londres et New York : Continuum, 2011.

Dans Les Naufragés et les Rescapés (1986), Primo Levi se souvient de la "brutale révélation " qui s'imposa à lui à son entrée à Auschwitz, que «l'espoir d'une solidarité de la part des compagnons d'infortune » était une grande illusion. L'absence de ce soutien attendu « devint 
évidente dès les premières heures d'enfermement, souvent sous la forme immédiate d'une agression concentrique de la part de ceux en qui on espérait trouver des alliés à l'avenir... » : «les premières menaces, les premières insultes, les premiers coups ne vinrent pas des SS mais d'autres prisonniers, de "collègues", de ces personnages mystérieux qui pourtant portaient la même tenue rayée qu'euxmêmes, les nouveaux arrivants, venaient d'enfiler.» La vie à l'intérieur du camp "ne pouvait pas être réduite aux deux blocs opposés des victimes et de leurs bourreaux", non seulement parce que les prisonniers s'exploitaient mutuellement, mais aussi parce que pour diverses raisons, certains d'entre eux obtenaient de la part de leurs geôliers des récompenses ou des privilèges. Pour Levi, c'est « la zone grise », l'espace qui se trouve entre les victimes et les bourreaux, peuplé de figures obscènes et pathétiques, où parfois, mais pas toujours, il est impossible de porter un jugement. Et alors qu'il réfléchit sur cet espace moral, il exprime l'évidence même : les rescapés "ne sont pas forcément toujours les meilleurs, ceux qui sont prédestinés à faire le bien, ceux qui portent un message », mais aussi «les égoïstes, les violents, les insensibles, les collaborateurs de la "zone grise", les espions $»^{2}$.

2 La zone grise de la Grande Famine ${ }^{3}$, c'est un monde à part peuplé de soupers ${ }^{4}$ et de grabbers ${ }^{5}$, de ceux qui prêtaient sur gages et de ceux qui vendaient la nourriture à des prix rédhibitoires, de ceux qui parmi les pauvres avaient une marmite pleine alors que leurs voisins mouraient de faim, ou encore des brutes qui dans les hospices volaient leurs biscuits aux plus faibles. C'est dans ce monde que l'on peut croiser une mère qui refuse de nourrir un enfant pour favoriser l'autre, un garçon qui tranche la gorge de deux jeunes pour un sac de nourriture, ou même des rumeurs ou des récits de cas de cannibalisme ${ }^{6}$. Et pour l'historien, qui doit se contenter des plus petits restes, c'est dans cet espace moral que vivaient Mary Gallagher et ses assaillants, qui pourraient bien être de sa famille, et qui pour ce qu'on en sait n'avaient peut-être pas suffisamment de pommes de terre pour eux-mêmes. En faisant ces remarques, on n'oublie pas ce que Margaret Kelleher appelle le risque d'une comparaison banale entre Famine et Shoah, pas plus qu'on ne commet de classification de la première de ces horreurs. Il s'agit plutôt de mettre en avant certaines difficultés posées par la condition à laquelle fut réduite par endroits cette humanité en haillons de l'Irlande des années 1840 , et de faire la lumière sur des situations pour lesquelles il n'était peut-être pas impossible, mais certainement pas aisé, de porter un jugement moral à l'époque, et il serait tout à fait déplacé de le faire aujourd'hui. Et pourtant, l'historien n'en a pas moins pour tâche de rendre compte d'une humanité réduite à cette condition et d'en attribuer les responsabilités.

Dans ses souvenirs sur la vie et la mort dans le Nord du Donegal du mi-dix-neuvième, Hugh Dorian fait preuve d'une conscience aiguë de cette zone grise ${ }^{7}$. Né en 1834 dans une petite ferme, il était tout jeune adolescent pendant la Famine, au moment où plus d'une personne sur quatre devait disparaître dans son entourage élargi ${ }^{8}$. En 1889-90, il écrivit au sujet de ce qu'il avait vu. Quatre décennies s'étaient écoulées depuis la Famine. C'est à peu près le même laps de temps qu'entre la Shoah et la rédaction de Les Naufragés et les Rescapés. Quoique moins bien calibré et moins cohérent dans ses jugements, mais aussi d'une force et d'une concentration intellectuelle moindres comparées à celles de Primo Levi, les mémoires de Dorian n'en demeurent pas moins le témoignage réfléchi de quelqu'un qui a survécu à une calamité épouvantable. Tout comme le survivant des camps de Si c'est un homme (1947), le survivant de la Famine se pose ces questions essentielles :

Les questions s'imposent à vous : qu'est-ce que l'homme? Et qu'est-ce que la vie? Qu'est-ce qui a bien pu causer tout cela ? Est-ce un châtiment pour nos crimes ? Ou pour les crimes de nos ancêtres, ou bien s'agit-il de purifier nos âmes ici-bas par la souffrance, pour les préparer à une éternité de bonheur dans l'au-delà ?⿳9 
4 Dorian ne se montrait pas désintéressé de la question des responsabilités de cette horreur à laquelle il avait survécu. Le plus souvent, l'attribution de ces responsabilités lui paraissait simplement évidente. S'il ne va pas jusqu'à accuser le gouvernement britannique d'avoir prémédité un assassinat de masse (une accusation que portaient certains nationalistes et républicains, mais absolument pas tous), il place toutefois les souffrances de cette période dans la lignée d'une longue histoire d'oppression coloniale, dont la violence était inscrite jusque dans le paysage, avec d'un côté « le terrain prospère de la plantation » et de l'autre « la montagne, la tourbière et le littoral », habités par " le Celte ». " Le paysan du Donegal », écrit-il, « a toutes les connaissances historiques nécessaires : l'histoire de ses ancêtres se déroule sous ses yeux chaque matin, exhibée dans chacun des éléments pittoresques du paysage (l'océan, la montagne, et son propre état de pauvreté) et s'il lisait quoi que ce soit, il faudrait que cela porte sur les raisons et les explications de sa présence en ce lieu. ${ }^{10}$ De plus, il tient bien la Grande-Bretagne pour responsable de la calamité et présente l'organisation de travaux publics comme le moment où

les conseillers du gouvernement ont asséné le coup de grâce - et cela peut paraître prémédité - ce coup qui devait faire lentement disparaître la vie humaine, pour se débarrasser de la population, rien de moins, en forçant des hommes affamés et à moitié nus à rester debout dans le froid, sous le grésil et la pluie du matin au soir, pour une récompense dérisoire de 9 pennies par jour. Si ces pauvres créatures pathétiques avaient reçu cette allocation directement chez eux, alors si petite qu'elle fût c'eût été un soulagement, ce serait de la charité, cela donnerait l'impression que leurs bienfaiteurs désiraient sauver des vies. Mais accordée de cette manière, sous conditions restrictives, c'était à la limite du meurtre à petit feu. 11

Toutefois, il est crucial de préciser que cette analyse nationaliste encadre une histoire toute personnelle. En effet, par moments la voix de Dorian résonne en dehors de la zone grise :

En conséquence de la mort, de l'émigration, et de la dispersion aux quatre vents, la population diminua rapidement. Et j'espère vraiment que ce n'est pas manquer de charité que de dire [cela, mais] au milieu de tout ça on a vu disparaître de nombreux personnages remuants et médiocres, qui ne faisaient pas honneur aux bonnes gens, aux honnêtes gens et aux bienfaisants, et si nous étions pauvres, nous avions aussi la paix. ${ }^{12}$

Ces paroles sont d'autant plus déstabilisantes que Dorian lui-même but et se bagarra tout au long de sa vie adulte, et que par une nuit de la fin décembre 1899 sa femme Catherine se noya après être tombée dans la Foyle, à Derry. On venait de la laisser quitter un poste de police de Strand Road pour trouver quelqu'un qui vienne chercher sa fille, arrêtée avec elle pour ébriété sur la voie publique ${ }^{13}$. Et si cette attitude " peu charitable » vis-à-vis de la perte de quelques-uns des membres les plus frustes de sa propre communauté dérange Dorian dans sa conviction que la Grande-Bretagne était responsable (à long terme comme à court terme) de la tragédie qui toucha l'Irlande, c'est aussi le cas de sa façon de s'accrocher à des explications providentielles de la faim et de la maladie, de la mort et de l'émigration :

S'il nous était permis de moraliser, nous dirions que le Tout-Puissant dans Sa grande sagesse a apporté la dispersion, l'émigration et la mort pour punir la population, trop nombreuse, trop indisciplinée et [...] trop rebelle dans son mode de vie ; c'est pourquoi il fallait un Pouvoir Supérieur pour la contrôler et la châtier. L'émigration en réduisit le nombre et les décima, mais donna à ceux qui restèrent, même peu nombreux, l'occasion de penser par eux-mêmes. ${ }^{14}$ 

s'ils étaient d'une autre espèce $»^{15}$, mais tous ne se ressemblaient pas et ne méritaient pas un tel traitement. Certains d'entre eux étaient des gens bien, honnêtes et bienfaisants, quand d'autres étaient remuants et médiocres. Quelques-uns avaient plus de nourriture que d'autres, plus d'influence sur les commerçants ou les membres des comités d'aide aux victimes, et d'aucuns se sentaient simplement moins liés par les valeurs de leur peuple. Par conséquent, quelques-uns recevaient la soupe populaire, d'autres utilisaient leur « influence » pour accéder aux travaux publics, et plus souvent, certains refusaient aux autres la charité de rigueur. Tout comme Primo Levi, Dorian insiste sur l'absence de solidarité entre ces "compagnons d'infortune ». "On oubliait l'amitié », écrit-il, "les hommes vivaient dans la peur de l'autre, chacun s'efforçant de s'en sortir au mieux tout seul, et quand un homme possédait des biens, il préférait le nier plutôt que de le faire savoir. " ${ }^{16}$ Si bien qu'un vieillard avec suffisamment de nourriture pour lui-même, mais trop peu pour la partager avec son petit-fils, devient lui-même une figure qui éveille la compassion ${ }^{17}$.

7 Si Dorian fait preuve de compassion envers un homme qui peut se nourrir lui-même mais pas sa famille, il n'en montre aucune pour ceux qui cherchèrent à tirer profit de leurs compagnons d'infortune. «Les propriétaires terriens sont souvent critiqués pour leur oppression, leur cruauté et leur tyrannie, mais malheureusement, un simple voisin peut bien souvent s'avérer être un tyran tout aussi cruel... " La bête noire ${ }^{18}$ de Dorian, c'est le grabber ${ }^{19}$, celui qui s'empare des terres d'autrui ${ }^{20}$. On pourrait considérer les baillis, qui favorisaient les grabbers, comme "choisis parmi la racaille de la pire espèce, sans scrupules et sans pitié ». Mais selon Dorian, on peut comprendre au moins une partie d'entre eux (assoiffés de vengeance et de la volonté d'imiter leur maître, facilement corruptibles avec nourriture et boisson) car ils ne sont qu'humains, avec leurs faiblesses :

Il est vrai que la plupart de ces glandeurs de baillis ont toujours été la lie de l'humanité, mais quelques-uns étaient forcés d'accepter ce travail peu glorieux, poussés par le souvenir d'une oppression ou d'une cruauté qu'eux-mêmes ou leurs proches avaient subie peu de temps auparavant. Ils avaient donc des comptes à régler, et ne pouvaient le faire qu'en se mettant sous la protection du propriétaire terrien, soutenus par son pouvoir. Alors, le fermier pour qui détruire une cahute ne signifiait rien, s'apercevait avec chagrin que l'insecte qu'il avait écrasé n'avait pas été détruit, et que celui qu'il méprisait tant était désormais en mesure de le persécuter, de le tourmenter, et à terme de l'expulser. Si la cruauté passée peut justifier l'esprit de vengeance, il est à craindre que certains n'ont pas oublié. Ainsi va l'humanité, et il n'est pas encore venu le temps où les hommes oublieront leurs blessures et rendront le bien en retour. ${ }^{21}$

Mais les grabbers sont eux-mêmes consumés par une avidité inhumaine. Et à travers son insistance répétée sur leur extraordinaire avarice - "l'homme avide réfléchi profondément, ses yeux voient loin » (restitution du proverbe irlandais Tchí saint $i$ bhfad: "l'avidité voit loin $)^{22}$ - il met sans doute le doigt sur une règle en matière de famine : en ces temps de grande pénurie, ceux qui ont le plus de chances de s'en sortir, ce sont les « avides » parmi les pauvres, les premiers à cesser de respecter la coutume du partage. Il est révélateur de constater que dans sa description des événements dans sa propre communauté, Dorian fait le parallèle entre l'ascension des grabbers (notamment « ceux que l'on pourrait qualifier de "classes inférieures" ") et la chute "des "bonnes gens" [...] qui se refusaient le moindre dérapage et ne voulaient pas être associés à la moindre mauvaise action. ${ }^{23} \mathrm{Et}$ si Dorian anticipe ici la crainte de Primo Levi que «les pires [...] les égoïstes, les violents, les insensibles, les collaborateurs » prédominent parfois parmi les survivants, alors on peut en prendre pour 
preuve l'indifférence absolue que lui-même exprime à l'égard de la disparition de ses voisins « remuants et médiocres».

Hugh Dorian mourut dans une maison surpeuplée de Nelson Street, dans le Bogside de Derry, et depuis 1914 il repose dans une tombe anonyme du cimetière de Derry City. Jamais la ville de Derry ne lui érigera de monument. Et d'ailleurs, quelle ville rendrait hommage à un homme qui reste indifférent à la mort de «beaucoup » de ses voisins? Mais le témoignage de Dorian est exceptionnel. Aucun autre membre de la classe populaire n'a couché sur le papier un compte-rendu aussi terre-à-terre et complet de l'horreur qui a fait naître l'Irlande moderne. On a toutefois entendu l'écho d'autres récits de la Famine jusqu'au milieu du vingtième siècle. Principalement, lorsque les collecteurs de l'Irish Folklore Commission demandèrent à des gens nés quelques dizaines d'années après la Famine ce que leurs parents leur avaient dit à propos d'am an drochshaoil [«le temps du mal vivre»], leurs récits fourmillaient de personnages pratiquant le vol ou le recel, récupérant la terre d'autrui, marchandant les repas ou prêtant sur gages. Parfois, ils mettaient même des noms sur ces figures "obscènes et pitoyables" de leur propre communauté (des gens dont les descendants vivaient encore tout près de chez eux), et de temps en temps ils discutaient de situations sur lesquelles il vaut mieux s'abstenir de porter un jugement. Les scènes se déroulant autour d'une marmite pendant la Famine sont une préoccupation majeure de la seanchas ${ }^{24}$. Par exemple, à Rann na Feirste, dans l'Ouest du Donegal, Séamas ó Dónaill, né vers 1858, se rappelait avoir entendu dire que lorsqu'une marmite ou une casserole de bouillon était sur le feu dans la deuxième année de la Famine, deux femmes des alentours qui se chargeaient de distribuer les rations servaient d'abord le gruau présent sur le dessus pour se garder le gríodar ${ }^{25} \mathrm{du}$ fond, plus épais et nourrissant.

Is iomaí lá garbh a bhí ag an choire chéanna. Bhí na daoine chomh hocrach agus nár fhan thuaigh nó grásta ar bith iontu. ... Il y eut de nombreuses journées difficiles autour de la même marmite. Les gens avaient tellement faim qu'ils n'avaient plus de pitié, ni de retenue. Un homme fort écartait les femmes, les enfants et les faibles de son chemin. Ils se marchaient les uns sur les autres, pour tenter de se rapprocher de la marmite.

Un jour, un pauvre homme se rendit à la marmite, et demeura un moment à l'écart de la foule, qui était tellement dense qu'une femme perdit connaissance. On s'écarta pour laisser passer deux hommes qui la lâchèrent sur le sol, derrière la foule. Puis ils s'en retournèrent vers la marmite, sans se soucier de savoir si cette femme était encore en vie. En arrivant, ils trouvèrent le pauvre homme qui avait pris la place de la femme à côté de la marmite. L'un d'eux l'attrapa à la gorge et le balança sur un tas de pierres qu'il heurta avec l'arrière de son crâne.

«Il en faudrait peu pour qu'on te mette dans la marmite», dit-il au pauvre. «Dire que tu viens ici boire notre bouillon quand il y en a bien assez peu, trop peu, pour nous seuls".

Le pauvre homme se redressa et s'éloigna sans dire le moindre mot.

Un homme du nom d'Eoghan Thuathail était présent à la marmite. Il avait observé toute la scène et fut choqué des mauvais traitements infligés au pauvre homme. Il le rappela pour lui offrir la moitié de son bouillon ${ }^{26 .}{ }^{27}$

10 La zone grise de la Famine (et la violence des pauvres envers les pauvres qui en est au cœur) est moins présente dans les histoires de ces années qu'elle ne l'est dans la seanchas ${ }^{28}$ . De tels ouvrages ont longtemps été rares. En effet, de la publication de Great Hunger de Cecil Woodham-Smith en 1962 (un best-seller international traduit de l'anglais par André Tranchant sous le titre La Grande Famine d'Irlande, 1845-1849 et publié chez Plon en 1969), jusqu'au cent-cinquantenaire de cette crise au milieu des années 1990, il n'y eut que deux 
tentatives d'un récit général de la Famine : un pamphlet de Mary E. Daly, The Famine in Ireland (1986), que Cormac Ó Gráda décrit généreusement comme " avare d'émotion dans les descriptions $»^{29}$ et Paddy's Lament: Ireland, 1846-47: Prelude to Hatred de Thomas Gallagher (1982), que l'on pourrait qualifier, dans le même esprit, d'extrêmement généreux en la matière. L'une était une révisionniste assumée, et l'autre mettait tellement d'enthousiasme dans son nationalisme que les historiens n'ont pas jugé bon d'en discuter sérieusement. Le cent-cinquantenaire a poussé plusieurs auteurs à se frotter à l'exercice d'une histoire générale, ce qui a entraîné un certain nombre d'ouvrages utiles, aux ambitions et aux approches diverses, destinés à un lectorat limité. Parmi eux, on trouve le superbe The Irish Famine de Peter Gray (1995), disponible en français chez Gallimard sous le titre L'Irlande au temps de la Grande Famine, mais aussi A Death-Dealing Famine de Christine Kinealy (1997), plus complet que son ouvrage précédent qui portait sur l'administration de la Poor Law, et The Great Irish Potato Famine de James S. Donnelly Jr., qui représente un ensemble cohérent basé sur une recherche approfondie et originale, même s'il comprend des versions revues d'essais déjà publiés (qui remontent pour la plupart jusqu'à 1989). On pourrait ajouter The Great Shame, de Thomas Kineally (1998), dont une partie importante est consacrée à la Famine, un sujet qu'il a de nouveau abordé dans son dernier ouvrage : Three Famines: Starvation and Politics (2011). Il y compare l'Irlande du mi-dix-neuvième siècle avec le Bengale du mi-vingtième et l'Éthiopie de la fin du vingtième.

11 La plupart des ouvrages du cent-cinquantenaire (et quelques histoires locales ambitieuses parues à peu près au même moment), commencent par présenter l'État, la société et l'économie du début du dix-neuvième siècle, avant de passer à l'impact du mildiou, aux différentes aides mises en place par le gouvernement ou des agences non gouvernementales, puis, enfin, aux évictions, à la mortalité et à l'émigration. C'est toute une histoire, qui intéresse des disciplines diverses, et si ce sont les pauvres que l'on visualise en la racontant, les voix qui se font entendre le plus souvent sont celles des politiciens, des hommes de presse ou des philanthropes, celles des officiels et des propriétaires terriens. Et c'est bien dommage, car l'expérience irlandaise du colonialisme et du nationalisme culturel a, entre autres choses, fait l'objet de deux sources d'archives différentes pour les historiens du dix-neuvième et du vingtième siècle, qui n'ont pas leur pareil ailleurs dans le monde. La première, c'est l'immense archive de comptabilité sociale, conservée principalement à Dublin Castle, et la seconde ce sont les enregistrements de l'Irish Folklore Commission, déjà mentionnés. Seule la Folklore Archive of Finland est en mesure de rivaliser en matière d'histoire orale dans une société agraire. De cette façon, il est possible d'apercevoir ce qui se passait derrière les fenêtres des casernes, et de percer à travers la fumée de tourbe des cahutes de terre. Avec l'aide de ces deux sources essentielles (et de quelques autres), les historiens de l'Irlande du dix-neuvième siècle ont la possibilité de produire une histoire sociale et culturelle de la plus grande qualité, qui pourrait permettre une appréhension plus précise de la Famine, et de son héritage.

Depuis les années 1990, la zone grise de la Famine s'est éclaircie. Si les figures pitoyables et obscènes ont toujours été bien présentes dans les sources écrites (comme en témoigne la description de la mutilation de Mary Gallagher), on en a toutefois une conscience plus aiguë, et elle est mieux connue grâce aux deux compilations d'histoire orale de Cathal Póirtéir: Famine Echoes (1995) et Glórtha ón Ghorta (1996) ${ }^{30}$, ainsi qu'à l'ouverture des deuxième et troisième cycles d'éducation supérieure dans les dernières décennies du vingtième siècle, qui a permis à des descendants de paysans pauvres de devenir 
historiens. Les historiens se sont penchés plus précisément sur le souperism ${ }^{31}$ pendant la Famine, un sujet qui avait déjà fait l'objet de quelques ouvrages. L'histoire de la prévention se développe également, et plus récemment celle du cannibalisme. Sur ces deux derniers sujets (comme sur beaucoup d'autres), les travaux de Cormac ó Gráda sont précurseurs, et ils sont bien partis pour faire référence encore longtemps. Et pourtant le grabber $^{32}$, le méchant par excellence de la seanchas ${ }^{33}$, n'a lui toujours pas d'histoire. On connaît depuis longtemps les statistiques moyennes (concernant par exemple le déclin du nombre de petites fermes) qui permettent d'évaluer les larges étendues de terre qui changèrent de main. Dans son livre écrit voilà 41 ans, Ireland Before the Famine, toujours d'un grand intérêt et toujours disponible, Gearóid ó Tuathaigh fait remarquer que les lopins de moins de 2 hectares représentaient $44,9 \%$ de toutes les fermes d'Irlande en 1841, mais seulement $15,5 \%$ en 1851. D'après Ó Tuathaigh, les chiffres "parlent d'euxmêmes. $\|^{34}$ C'est effectivement le cas, et ils posent les questions suivantes : À qui la terre est-elle revenue? Les données confirment-elles cette idée répandue de pauvres récupérant la propriété de gens plus riches, et si c'est le cas, comment cela fut-il possible? Dans quelle mesure le profiteur est-il une invention du milieu des années 1850, au moment où la brusque remontée des prix fit regretter leurs transactions à ceux qui s'étaient débarrassés de leur terre dans cette mauvaise passe ? Les propriétaires terriens, et même l'État (à travers la Poor Law) étaient-ils complices de l'agrandissement des fermes de quelques-uns aux dépens des autres?

Exception faite du mystérieux grabber ${ }^{35}$, on comprend mieux aujourd'hui l'expérience des pauvres qu'on ne la comprenait dans les années 1990, et peut-être peut-elle désormais, dans toute sa misère grisâtre, occuper une place centrale dans l'histoire de la Famine. Et pourtant, le travail des historiens ne consiste pas qu'à accumuler les types de personnages. Dans un essai lumineux du milieu des années 1990, qui aujourd'hui encore vaut d'être lu, Colm Tóibín examinait les documents sur la Famine recueillis par l'Irish Folklore Commission, qui commençaient tout juste à être dépoussiérés par Póirtéir, Ó Gráda, Patricia Lysaght, Niall ó Ciosáin et quelques autres :

On peut faire ce qu'on veut des archives folkloriques sur la Famine: on peut les considérer comme emplies de légendes et de contes, on peut en faire une mine d'informations à traiter comme une source primaire, elles représentent une histoire de la mémoire irlandaise après 1935, elles devraient être consultées par tous les historiens qui écrivent sur la Famine, et par les critiques littéraires.

C'est toutefois une mine d'informations inestimable. Elle est aussi parsemée de pièges, comme le montre cette réponse à la question 2 d'un habitant de Boyle, dans le comté de Roscommon, à propos d'un enterrement pendant la Famine : «Certains cadavres étaient simplement enveloppés dans un drap et enterrés. On mettait parfois de nombreux corps dans une même tombe. Personne ne voulait s'approcher des corps, de peur d'attraper la fièvre. À certains endroits, où la fièvre avait fait moins de ravages, les gens ont vu des cercueils flotter. »

Comment prendre les trois premières phrases comme un témoignage sérieux des rites funéraires pendant la Famine, alors que c'est impossible pour la dernière? Cette dernière affirmation ne neutralise-t-elle pas les trois précédentes? Mais elle nous aide à analyser le fait que la fantaisie gothique n'est jamais loin dans les récits de la Famine. Et les trois premières sont confirmées par d'autres récits. ${ }^{36}$

14 Ce qui semble implicite ici, c'est l'intuition d'un romancier que les formes narratives habituelles doivent être outrepassées pour que les historiens puissent espérer expliquer la Famine. À propos du travail de maître de Woodham-Smith, Tóibín considère que « plus jamais personne ne pourra écrire de cette manière. Lire The Great Hunger, c'est comme lire de la poésie de l'époque géorgienne tout en sachant qu'une nouvelle forme de poésie, moderne et 
décomposée, se prépare. $\|^{37}$ À mon avis, les récits "modernes " garderont longtemps une place de choix, en particulier les simples introductions ou les enquêtes, mais la réalité comme l'imagination des pauvres de la mi-dix-neuvième s'éclaircissent (en particulier ces personnages obscènes et pitoyables de la zone grise), et une nouvelle manière de raconter, plus ambitieuse et plus risquée, pourrait devenir nécessaire, si l'époque où l'on voyait flotter des cercueils devait à nouveau faire l'objet d'un « grand livre ».

\section{Quelques histoires}

Quatre histoires de la Famine ont paru ces trois dernières années. À l'exception de Ciarán Ó Murchadha, qui avait déjà publié une belle étude de la Famine dans le West Clare, il est intéressant de noter qu'aucun des auteurs n'a beaucoup de "pratique" sur ce sujet. Jusqu'à présent, Enda Delaney se consacrait à l'histoire des Irlandais en Grande-Bretagne au vingtième siècle, et John Kelly a écrit un ouvrage reconnu à propos d'une autre grande catastrophe européenne, plus ancienne : la Grande Peste. Enfin, après avoir été un grand journaliste, Tim Pat Coogan a écrit des ouvrages d'importance sur De Valera, Collins et l'IRA, ainsi qu'un autre livre, On the Blanket (1980), un appel tardif à la résolution des crises de Long Kesh et Armagh $^{38}$, qui n'eut malheureusement pas suffisamment d'influence.

Des quatre ouvrages, c'est celui de Tim Pat Coogan, The Famine Plot, qui a le plus attiré l'attention, souvent à ses dépens. On imagine que "Quarante nuances de Vert " $^{39}$ conviendrait davantage à Coogan que Cinquante nuances de Grey et c'est sans aucun doute la forte présence du vert plutôt que l'absence du gris (au sens de nuance intellectuelle ici) qui a stimulé au moins un de ses critiques : le grand contradicteur de Queen's University à Belfast, Liam Kennedy. Le livre de Coogan témoigne d'une des interprétations nationalistes de la Famine: celle selon laquelle la Famine était un acte génocidaire prémédité de la part du gouvernement britannique. Une fois de plus, la thèse du génocide n'a jamais été la seule interprétation nationaliste, et il est malhonnête de la part des critiques de Coogan de la présenter comme telle. Toutefois, il suffirait d'une pointe de vert pâle, d'un simple sous-entendu laissant penser que la Grande-Bretagne n'a pas toujours eu un rôle positif et progressiste en Irlande, pour réveiller quelques-uns des membres de l'infanterie révisionniste, qui continuent à se battre dans la jungle historiographique des années 1970 et 1980. Et Coogan utilise ici un tube de «vert John Mitchell $»^{40}$ tellement vif, qu'il ne pouvait que déclencher des coups de tonnerre.

Mais il s'est écoulé pratiquement un quart de siècle depuis 1989 et l'argument de Cormac ó Gráda selon lequel «aucun historien digne de ce nom ne prend plus au sérieux le moindre argument en faveur du "génocide" $»^{41}$. The Great Famine: Ireland's Agony, 1845-52 de Ciarán Ó Murchadha, chaleureusement défendu par ó Gráda (et avec raison) comme un "travail d'une grande puissance narrative et analytique [...], accessible, courageux, et habilement écrit ", se termine par l'idée qu'il faudrait toutefois continuer à en débattre :

La question de savoir si l'on peut ou non considérer le comportement du gouvernement britannique comme génocidaire dépend en grande partie de la définition qu'on en fait. Depuis le milieu du vingtième siècle, le terme de génocide est si étroitement associé à l'holocauste perpétré par les nazis, que son sens s'en est trouvé modifié et limité. Et si l'on voit le génocide comme la destruction délibérée et systématique de tout un groupe ethnique ou religieux par un assassinat de masse, nulle part on ne trouve aucun équivalent au dix-neuvième siècle. Toutefois, 
si on le définit comme l'utilisation délibérée et systématique d'une catastrophe environnementale pour détruire un peuple sous prétexte de mettre en place une réforme sociale, alors il y a matière à discussion. est si près que cela ne change pas grand-chose. Et il faut préciser également que sa réflexion sur le génocide (tout comme celle sur la représentation insulaire de la Famine comme « un crime contre l'humanité particulièrement odieux, commis à l'encontre d'un peuple sans défense ») ressemble fort à une arrière-pensée qui se révèle dans sa conclusion, subitement et sans prévenir. Autrement dit, il ne construit pas dans son livre d'argumentation claire en faveur de la thèse du génocide. De plus, tout en assurant que cette "accusation» dépend des définitions, à aucun moment ó Murchadha ne fait référence à la recherche en droit. C'est étonnant, car la National University of Ireland, Galway, où il a obtenu son doctorat, peut s'enorgueillir d'avoir un ponte en la matière : William Schabas ${ }^{42}$. Et dans son avant-dernier paragraphe, il propose une paraphrase déformée de la Convention des Nations Unies pour la prévention et la répression du crime de génocide (1948) et une citation erronée de la fameuse sentence de John Mitchel : « Certes le Tout-Puissant nous a envoyé le mildiou, mais ce sont les Anglais qui ont créé la Famine " (il a remplacé le verbe "créer » par « envoyer »). Tout cela pousse à penser que cette ouverture est une addition tardive et précipitée à la toute fin du livre, la conclusion hâtive d'un ouvrage attentif à l'écriture solide ${ }^{43}$.

Mais le problème le plus pressant, c'est que le «Gros mot $»^{44}$ (l'euphémisme qu'utilisent les diplomates américains pour éviter de prononcer un mot qui les mettrait dans l'obligation légale, morale et politique d'agir contre ce qu'il décrit ${ }^{45}$ ) a ainsi été mis en jeu. Il y a désormais des « historiens dignes de ce nom » comme ó Murchadha qui veulent bien « prendre au sérieux » l'idée que quelques-unes des politiques imposées à l'Irlande à la fin des années 1840 pourraient être considérées comme génocidaires. Et d'autres historiens défendent l'idée opposée. Mais, dans la mesure où un « débat » existe, il reste décevant. Pour commencer, si la plupart des participants au débat (qu'ils soient ou non des universitaires, qu'ils dénoncent le génocide ou qu'ils le nient) utilisent des références symboliques à la Convention des Nations Unies, aucun n'a jamais fait preuve d'une appréhension plus que profane des lois concernant le génocide. Voilà un sujet sur lequel les spécialistes du droit ont énormément écrit et débattu, en particulier sur la question de l'usage précis du terme lui-même. Si (et ce «si » là est d'une importance phénoménale) on veut débattre de ce qui s'est passé en Irlande dans les années 1840 en faisant référence aux lois du vingtième siècle, alors ne faut-il pas faire preuve d'une connaissance suffisante de la loi, de la recherche en droit et des questions qu'elle a soulevées? À l'écoute d'une émission de radio dans laquelle Liam Kennedy et Coogan s'affrontent tels Judy et Punch à propos des «intentions» et de "preuves irréfutables» (un entretien que Kennedy lui-même a décidé de transcrire pour le publier dans la Dublin Review of Books : < http://www.drb.ie/blog/writers-and-artists/2013/02/25/was-the-famine-a-genocides)

on a l'impression qu'aucun des deux ne connait grand chose à la législation internationale ${ }^{46}$. Par ailleurs, et cela a nettement plus d'importance, aucun historien n'a remis en cause la pertinence d'un jugement porté sur le passé à partir de lois créées plus tard, ni même averti des risques de ce que Carlo Ginzburg appelle « une historiographie moraliste basée sur un modèle judiciaire ${ }^{47}$. Si seulement un historien irlandais avait depuis longtemps paraphrasé le plaidoyer exaspéré de Marc Bloch aux historiens de la Révolution Française : «Robespierristes, antirobespierristes, nous vous crions grâce; par pitié, dites-nous simplement : qui était Robespierre ? $»^{48} \mathrm{Si}$ seulement, c'est-à-dire, quelqu'un avait dit, depuis 
longtemps : « Vous qui dénoncez le génocide, vous qui le niez, nous vous crions grâce : par pitié, dites-nous simplement comment la Famine fut vécue par les paysans pauvres et les travailleurs, par les petits fermiers et les artisans. » Bien entendu, tout historien qui défendrait une telle position aujourd'hui (celle qui consiste à dire que l'histoire vise à comprendre ce qui s'est passé, pas à juger) serait probablement tout autant décrié par chacun des deux camps pour avoir joué les insoumis, même s'il serait probablement plus approprié de lui reprocher de jouer les objecteurs de conscience. Et il est vrai que la différence entre le juge et l'historien, comme Ginzburg lui-même le concède, n'est pas aussi nette que Bloch la représentait. Les étudiants de troisième cycle avait pour habitude de se demander si Bloch (s'il n'avait pas été tué par la Gestapo en 1944) aurait jugé Hitler, ou s'il aurait tenté de le comprendre. Aujourd'hui, beaucoup d'historiens soulignent le fait que cette insistance de Bloch sur les dangers du jugement moral suppose une distance critique qui ne colle pas parfaitement avec son insistance justifiée sur l'impossibilité d'atteindre l'objectivité, ce "noble rêve", comme dit Peter Novick $^{49}$, que le critique Seamus Deane a décrit il y a longtemps comme le "pays imaginaire de l'imagination » des révisionnistes irlandais ${ }^{50}$.

Mais toutes ces questions n'ont jamais été intégrées au débat pour savoir si oui ou non les politiques individuelles mises en place en Irlande à la fin des années 1840 étaient génocidaires. En fait, les arguments avancés sont loin d'être aussi subtils : c'est «la Famine » elle-même qui est, oui ou non, « un acte génocidaire ». Au milieu de ce débat véhément, on ne trouve aucune réflexion sur les préoccupations adaptées au «travail de l'historien ", tout le monde ne fait que se triturer les méninges (souvent de façon très arrogante) sur le rôle que doit jouer l'historien dans la vie publique ${ }^{51}$. Quelle que soit la pièce de Yeats responsable des tirs anglais ${ }^{52}$, je doute que les chiffres donnés par Mary Daly à propos de l'exportation de nourriture aient empêché qui que ce soit de s'engager dans des organisations paramilitaires républicaines dans les années 1980. Autrement dit, on est en droit de supposer que ceux qui se sont ainsi engagés avaient trouvé pour cela des raisons suffisantes dans les conditions de vie de leur propre époque, avant de commencer à dépoussiérer John Mitchel. Et pour insister encore davantage sur ce point, le fait de considérer ou non la Famine comme un génocide ne retire ni n'ajoute rien aux arguments politiques de 2013 en faveur ou à l'encontre de la réunification ; c'est sans aucun doute ce qui préoccupe Kennedy, car la défense de la repartition ${ }^{53}$ fait partie des symptômes de sa chlorophobie aiguë (une aversion chronique du vert). Disons-le carrément: étiqueter certaines des politiques menées par le gouvernement à la fin des années 1840 comme "génocidaires» (ou même décrire «la Famine » comme un «acte génocidaire ») est devenu un problème dont la portée est tout autant légale que politique.

21 Kennedy s'est attribué une position fort peu enviable dans ce débat radiophonique, qu'il a, encore une fois, lui-même choisi de transcrire et de publier quelques mois plus tard dans la Dublin Review of Books. Face à l'idée de la Famine comme génocide, il a soutenu qu'elle n'en était pas un en s'appuyant sur la Convention des Nations Unies pour la prévention et la répression du crime de génocide, pour lequel il ne s'agit d'ailleurs pas nécessairement de tuer. Par exemple, l'article $2 . \mathrm{b}$ mentionne une «atteinte grave à l'intégrité physique ou morale » des membres d'un même " groupe national, ethnique, racial ou religieux» comme définissant le génocide, tandis que l'article 2.c ajoute la «soumission intentionnelle $d u$ groupe à des conditions d'existence devant entraîner sa destruction physique totale ou partielle». Et maintenant qu'il a rejeté cette "accusation» (en donnant l'impression d'un homme qui vit dans un monde très plat du point de vue du droit), que 
fera Kennedy si un chercheur en droit sérieux lit de la recherche historique sérieuse des vingt dernières années (comme par exemple le travail du collègue de Kennedy, Peter Gray), le rapproche des lois du vingtième siècle, et en arrive à une conclusion opposée ? Kennedy se réveillera-t-il subitement de son «noble rêve " pour découvrir le Bloch qui est en lui et nous expliquer que l'histoire consiste à comprendre, pas à juger? Ou bien s'achètera-t-il simplement un livre de droit, pour en discuter avec les spécialistes?

Au milieu des années 1990, Peter Gray argumentait comme suit :

On ne peut que condamner la politique mise en place en Irlande à partir de l'automne 1847 (malgré l'opposition de l'opinion publique irlandaise et l'avis négatif des administrateurs de l'île), puisqu'elle a fortement augmenté la misère du pays. On peut difficilement contredire l'accusation portée par un observateur anglais qui participait à l'aide humanitaire à la fin de la Famine et dit qu'au beau milieu "d'une nourriture abondante et bon marché [...] nombreux sont ceux qui ont été poussés vers la mort par pure tyrannie. " L'accusation de négligence coupable quant aux conséquences de politiques qui ont mené à une famine massive est indéniable. On peut aussi démontrer que plusieurs ministres ont fait en toute conscience le choix de faire passer des objectifs moraux et économiques avant la défense de la vie humaine.

Mais Gray ajoute également qu'il n'a trouvé aucune preuve d'une " politique génocidaire délibérée " " ${ }^{5}$. C'est à se demander ce qu'un spécialiste du droit ferait du raisonnement de Gray si, comme le suggère Kennedy, il devait décider si oui ou non les mesures imposées à l'Irlande dans les années 1840 étaient génocidaires avec pour référence la Convention des Nations Unies de 1948 et, comme on se doit de le supposer, les experts qui y ont contribué. Quel spécialiste pourrait lire l'ouvrage de Gray sans penser à la remarque introductive de Schabas selon laquelle «le génocide a le plus souvent été commis (même si ça n'est peut-être pas systématiquement vrai) sous les ordres de l'État dans lequel il a été commis, ou tout du moins avec sa complicité bienveillante $"^{55}$ ? De la même manière, que ferait un spécialiste du droit (qui suivrait les conseils de Kennedy et aurait en main la Convention des Nations Unies de 1948) de la Gregory Clause, qui à partir de 1847 exigeait des plus pauvres qu'ils abandonnent leur moyen de survie (à l'exception d'un millier de mètres carrés) en échange d'une aide immédiate. Ferait-il référence à l'article 2.c qui parle, répétons-le, de la "soumission intentionnelle du groupe à des conditions d'existence devant entraîner sa destruction physique totale ou partielle »? Liam Kennedy joue là une partie qui n'a pas grand chose à voir avec l'Histoire et qu'il ne peut pas remporter. Une fois de plus, sa chlorophobie a pris le dessus.

Les historiens ont beaucoup à apprendre des spécialistes du droit sur la façon de qualifier les décisions prises par le gouvernement britannique dans les années 1840, plus particulièrement sur la législation et le concept même de génocide, ainsi que sur l'échelle des responsabilités. Et il est possible qu'à l'avenir les historiens considèrent ó Murchadha (et pourquoi pas aussi Tim Pat Coogan) comme celui qui nous aura rendu à tous un grand service en nous sortant à la fois de la polémique nationaliste du dix-neuvième siècle et de la polémique révisionniste des années 1970 et 1980, c'est-à-dire en nous rendant libres (après une dernière discussion animée) d'utiliser avec parcimonie un mot dont les historiens se servent pour décrire des événements terribles ayant eu lieu ailleurs, donc d'avoir la possibilité de dire par exemple que « la Gregory Clause était génocidaire » sans avoir à dire que "la Famine " (un événement complexe qu'il est même difficile de périodiser) était « un acte génocidaire ». Pour ma part, si j'accepte tout à fait l'idée que l'historien ne se contente pas d'expliquer mais puisse aussi juger, l'enthousiasme avec 
lequel on étiquette un événement comme tout noir ou tout blanc me met mal à l'aise. Et au beau milieu de ces chamailleries sur comment étiqueter la Famine se cache une idée que Hugh Dorian, dont la vie fut dure, exprime bien, et qu'Enda Delaney, John Kelly et Ciarán Ó Murchadha ont bien saisi (et avant eux James S. Donnelly, J ${ }^{56}$ ). C'est l'idée que pour les pauvres, les politiques gouvernementales étaient « $\underline{\text { à la limite }}$ du meurtre à petit feu » et qu'à leurs yeux l'objectif qui «semblait prémédité" était de «se débarrasser de la population» (souligné par l'auteur). Les pauvres n'ont pas eu besoin de John Mitchel pour considérer l'État comme responsable de l'étendue et de la forme que prenaient leurs malheurs: ils étaient capables d'en arriver à cette conclusion par eux-mêmes, tout comme les membres de chacune des autres classes sociales. C'était en tout cas évident pour les habitants de Ballybofey, d'où une lettre intitulée «Starvation» (probablement pas rédigée par l'un des plus pauvres) fut transmise au Ballyshannon Herald qui la publia le 29 janvier 1847 :

MONSIEUR - Même en ayant de quoi payer, il était impossible de se procurer la moindre bouchée de nourriture à Ballybofey, ni lundi, ni mardi, ni mercredi. Les marchants comme les profiteurs assuraient ne rien avoir. Et aujourd'hui (jeudi, jour de marché), il y en a en abondance pour le prix de 2 shillings et 6 pence la bouchée. Cet exemple, comme d'autres, prouve à quel point il est absurde et inhumain de la part de Lord John Russell de faire confiance à la spéculation et à l'entreprise privée pour fournir de la nourriture aux foules affamées. Pour l'entreprise privée, quel est l'intérêt d'une nation qui crie famine? L'objectif de l'entreprise privée est d'accumuler l'argent dans les poches privées. Périr, si toutefois on peut atteindre cet objectif, voilà le vocabulaire de l'entreprise privée. Sans une intervention rapide et efficace de la part du Gouvernement, qui consisterait à envoyer de la nourriture en dehors de ce que la spéculation privée veut bien fournir au pays, les cantons peuplés qui dépendent de Ballybofey pour obtenir de la nourriture, et bien d'autres endroits dans la même situation, seront bientôt le nouveau Skibbereen. ${ }^{57}$

L'insistance de ce courrier sur l'idée que la responsabilité suprême de nourrir ceux qui n'avaient pas la possibilité de se nourrir par eux-mêmes revenait à l'État est représentative de la ligne politique adoptée à ce moment là par ce tout petit journal tonitruant qu'était le conservateur Ballyshannon Herald. Un paragraphe d'une seule phrase avait paru dans le numéro du 8 janvier : "À travers toute l'Irlande, les petits fermiers en tant que classe à part entière sont en voie de quasi disparition, et ce en raison de la visite de la famine, qui fait des ravages dans le pays. ${ }^{58} \mathrm{La}$ semaine suivante, un éditorial peignait les choses d'une manière plus vive encore :

Chaque jour, la situation des paysans et des ouvriers se détériore davantage. Nous pensons sincèrement que dans tout le comté, et dans cette région-ci en particulier, la majorité d'entre eux n'atteint pas une moyenne de trois repas par semaine. Ils font peur à voir, et malgré tout ils demeurent pacifiques et résignés, comme l'était Job. C'est un problème que de savoir ce qu'il va advenir d'eux! Si le parlement n'agit pas à grande échelle dès sa prochaine réunion, ils mourront dans les champs tels des moutons avariés. ${ }^{59}$

Ils étaient, clamait le journal à la fin du mois, «la population sacrifiée du pays $»^{60}$; il n'y avait rien d'autre à faire, lisait-on en février, que d'en appeler au Gouvernement «pour qu'il accélère les mesures d'aide aux victimes et qu'il sauve à tout prix la population affamée, sans quoi le pays ne sera plus qu'un vaste champ de tombes. ${ }^{61}$

Plus tard, en 1848, alors que les intendants de propriétaires terriens pourchassaient les paysans pour obtenir les arriérés de loyer et que les agents de l'État venaient récolter les taxes, on assista à certains endroits à une recrudescence de la résistance populaire. Et bien souvent, l'impression que l'État était responsable du sort des pauvres gens renforçait 
cette résistance, tout comme l'impression que l'État cherchait à se débarrasser de cette population. En mai 1848, la police confisqua plusieurs exemplaires du texte d'une ballade qu'un homme de Fermanagh chantait et vendait aux alentours de Ballyshannon. Il fut traîné devant un magistrat qui fit envoyer un exemplaire du texte incriminé à Dublin Castle, comme exemple de «l'opposition qui se poursuit à l'égard de la Poor Law ». Intitulée $A$ New Song, called, The Poor-House, la ballade dénonçait la gestion déplorable de l'hospice d'Enniskillen, en nommant les notables mis en cause. Elle représentait plus particulièrement la Poor Law comme un outil pour la transformation du pays, qui servait à forcer les habitants à abandonner leurs terres, donc à se débarrasser d'eux par tous les moyens. Et pour appeler la population à résister, cette chanson faisait référence à une " oppression" plus ancienne, ce qui pousse à la considérer comme influencée par une certaine vision de l'histoire irlandaise, selon laquelle la volonté de «se débarrasser » de la population n'était pas nouvelle ${ }^{62}$ :

Our homes are levelled through country and town,

There's scarce a small cabin at all to be found;

'Tis all their whole password when going to their door,

We're building a house for you to be sure.

We'll shortly get rid of you by and by,

Like pigs we will gather you into one stye.

The walls we'll build high without any mistake,

For fear over them you would chance to break. [...]

For there they'll be masters of every degree,

From porters to gatesmen, as plain you may see,

Every man their own station will fill,

To subdue those poor creatures without their will. [...]

Now, to conclude and finish my song,

I hope those poorhouses will not reign long;

The cess is so weighty they're going to rebel,

And banish them off to Connaught or $\mathrm{H}_{-} 11 .^{63}$

Pour en revenir à Famine Plot, de Tim Pat Coogan, si c'est la lueur de vert qu'il contient qui a attiré l'attention de certains critiques, c'est surtout l'absence totale de gris qui leur a donné le cœur d'attaquer. Malgré sa conclusion « verte», l'ouvrage de ó Murchadha (qui s'appuie sur une recherche d'une plus grande qualité et une écriture splendide) ne s'est pas attiré tant de foudres. C'est une cible moins accessible. À l'inverse, l'ouvrage de Coogan se précipite, il charge dans la jungle, tête baissée. En plus de nombreuses erreurs et inexactitudes concernant les faits, l'ancien rédacteur en chef de l'Irish Press peut grimacer à la vue de «Fina Fail » ${ }^{64}$, et de nombreuses autres erreurs typographiques. Et certaines de ces erreurs ont de l'importance. Lorsqu'il présente le soulèvement de 1798 dans son chapitre introductif sur l'histoire du pays, il cite Joseph Holt, qui aurait vu les Ancient Britons ${ }^{65}$ "cutting the haunches and thighs off the women for wearing green stuff petticoats » (p. 16, souligné par l'auteur), alors que la version de cette citation donnée par Ruán O'Donnell (un historien d'une grande méticulosité) dans son histoire du soulèvement dans le comté de Wicklow ${ }^{66}$ est la suivante : "cutting the haunches and thighs of the young women ${ }^{67}$. Les femmes en question auraient senti la différence. Mais surtout, on ressent de façon évidente la précipitation dans l'argumentation et la rédaction, cela fait l'effet d'avoir été écrit au fil de la plume. Pour paraphraser James S. Donnelly Jr., The Famine Plot permet de rappeler à tous les historiens spécialistes de cette période qu'ils feraient bien de préserver cette indignation morale des nationalistes irlandais face à l'étendue de ce que les Britanniques étaient prêts à tolérer ${ }^{68}$. 

de Ciarán Ó Murchadha se sont tous vus éclipsés par un ouvrage de moindre qualité. L'ouvrage de Kelly a reçu par avance les compliments des grandes figures du Parti démocrate que sont Bill Clinton et Kathleen Kennedy Townsend. Le grand spécialiste des politiques publiques qu'est Clinton comprend que "les décisions politiques ont empiré la situation" et il salue The Graves are Walking comme "un récit édifiant pour quiconque prendrait le risque d'une catastrophe (qu'elle soit humaine, économique ou écologique) au nom d'une victoire idéologique." De même, Kennedy Townsend (elle-même bien rodée en politique) considère la Famine comme "une leçon pour notre époque» et se montre particulièrement satisfaite de la manière dont Kelly « dépeint avec force détails affligeants ces dirigeants britanniques qui, victimes de leur ferveur religieuse et de leurs ceillères idéologiques, décidèrent d'inculquer aux Irlandais de bonnes habitudes de travail et de les débarrasser de leur dépendance vis-à-vis du gouvernement. » La jaquette du livre de Ó Murchadha affiche l'éloge chaleureux et mérité du grand maître de la recherche sur la Famine : Cormac ó Gráda. Sans aucun doute une approbation des plus réjouissantes, même si ce simple économiste n'a pas l'aura d'une Kennedy ou d'un Clinton. Mais il semble que Delaney (un ami, avec qui je m'apprête à publier une compilation d'essais) n'ait pas de contacts hauts placés, que ce soit dans le milieu universitaire ou politique. Les citations affichées sur la jaquette de son livre sont celles des acteurs de la période : Charles Trevelyan, secrétaire assistant du Trésor, le révolutionnaire républicain John Mitchel et John MacHale, fils d'un gérant de pub qui devint, pour "les pauvres et les exclus", un archevêque de Tuam à l'immense popularité. La citation du notable du Trésor laisse paraître exactement l'attitude envers la société contre laquelle Clinton avertit les politiciens d'aujourd'hui : «Bien que le processus de modifier de vieilles habitudes et de reconstruire la société sur de nouvelles bases prenne forcément du temps, des signes certains montrent que nous avançons d'un pas assuré vers l'objectif recherché.» Celle du républicain décrit le prix payé par le sang pour une telle attitude :

Parfois devant les cottages, je voyais des enfants tout jeunes appuyés contre une grille dans le soleil, car ils ne tenaient plus debout : ils n'avaient que la peau sur les os, étaient à moitié nus, leurs visages à la fois bouffis et ridés avaient une teinte vert pâle. Il était évident que ces enfants-là ne deviendraient jamais des hommes ou des femmes. Je voyais les serres de Trevelyan se refermer sur les entrailles de ces enfants : sa bureaucratie les mènerait à la mort, il leur avait préparé dans son laboratoire gouvernemental le poison du typhus.

Pour finir, celle de l'archevêque évoque l'épiphanie politique venue de ce spectacle : Le fléau, le terrible fléau qu'était l'Union législative ne peut désormais plus être nié. On en a pour preuve les tombes des morts et les formes squelettiques des vivants, qui sont des monuments à l'accusation de cette cruelle politique qui a systématiquement limité le peuple d'Irlande à une nourriture digne de celle qu'on trouve à l'état sauvage. Pour maintenir le luxe et le monopole venus d'ailleurs, on leur a interdit l'accès aux récoltes et au bétail abondants qui grouillent sur cette terre et sont le produit de leur propre labeur acharné et silencieux.

Ces trois livres s'intéressent tous au même lieu et à la même époque, pourtant chacun raconte une histoire différente, et la raconte différemment. Ó Murchadha propose une large enquête et fait preuve d'une impressionnante maîtrise des sources et de la recherche antérieure, en particulier le corpus grandissant des études locales. Il raconte une grande histoire (tout en s'efforçant de prendre en compte les variations locales et régionales), et il parvient à la raconter à sa manière. Par exemple, il identifie deux " échecs » qui sont d'importance pour la Famine : l'échec de la politique d'aide de l'État, mais aussi l'échec de la charité privée, qui jusque-là avait été mise au second plan par des 
histoires de donations faites par les Indiens Choctaw d'Oklahoma ou par d'anciens esclaves des Caraïbes, et surtout par les comptes-rendus des efforts fournis par la Société religieuse des Amis. ${ }^{69}$ Bien entendu, on sait identifier le début du phénomène de lassitude à l'égard de la Famine (et surtout le moment où cette lassitude s'intensifie en GrandeBretagne après que quelques jeunes gens ont établi en tirant des coups de feu dans un potager du Tipperary ${ }^{70}$ que «les Irlandais » méritaient ce qui leur arrivait) mais ó Murchadha accorde une plus grande importance à l'échec de la charité privée, et ce faisant (avec l'appui de travaux déjà effectués par Kinealy) ${ }^{71}$, il remet tout en place. Ou plutôt il remet les pendules à l'heure en mettant en avant une organisation qui n'est pas souvent mentionnée dans les histoires de la Famine, qui existe toujours, qui s'est montrée particulièrement active ces quatre dernières années, et qui sera probablement plus active encore à l'avenir - la Société Saint Vincent de Paul :

En ce qui concerne la campagne internationale d'aide montée par l'Église catholique (qui fut l'une des plus importantes de l'histoire), elle cessa lorsque la papauté se détourna des révolutions internationales de 1848 et ne fut plus à même de maintenir ses collectes internationales. Toutefois, malgré cela, la campagne de l'Église catholique fut la seule à perdurer jusqu'en 1850. À ce moment là, la seule organisation importante à poursuivre l'organisation de collectes était la Société Saint Vincent de Paul. Depuis de nombreux pays, ses membres continuaient à envoyer des dons, par le biais de " conférences » organisées en Irlande à l'intention des affamés. Discrets et persévérants, les travailleurs de Saint Vincent de Paul ceuvrèrent sans relâche jusqu'à la fin de la Famine, et encore après, en évitant toute reconnaissance ou publicité au delà de ce qui était nécessaire pour obtenir de nouveaux dons.

Ó Murchadha et son éditeur, Continuum, ont fourni un travail remarquable avec Ireland's Agony, y compris à travers l'attention portée à la sélection et à l'emplacement des illustrations, notamment des images tirées de L'Illustration: Journal Universel de 1854, qui n'étaient pas connues.

The Graves are Walking de John Kelly est une histoire de la Grande Famine décrite avec une grande vivacité par un grand maître conteur. Kelly est particulièrement doué pour les petites anecdotes (il prend plaisir à préciser le temps qu'il faisait, ce que les lecteurs irlandais sauront apprécier) et il a le don pour trouver la juste expression : «La langue et la culture irlandaises", écrit-il, "s'exilèrent dans les cahutes des paysans". S'il maitrise visiblement moins bien qu'ó Murchadha les études locales, il connait tout aussi bien les parutions les plus récentes de la recherche sur la Grande Famine, il a l'œil pour les détails, il sait qui citer et à quel moment le faire, et il propose un compte-rendu très agréable à lire des événements des années allant de 1845 à 1848. La critique implicite dans tout cela, c'est que comme les notables britanniques, il enterre la Famine un peu rapidement pour déplacer l'action principale vers New York, et ne consacre pas beaucoup de réflexion à l'impact de la Famine sur la société, la culture et la politique irlandaises. Autrement dit, il ne transmet pas vraiment le sentiment que c'est de l'horreur et de la misère qu'a émergé l'Irlande moderne, ce qu'ó Murchadha, lui, fait à merveille, et aurait pu faire encore plus en longueur. Cependant, c'est clairement le public américain qui est visé par The Graves are Walking, et l'ouvrage pris sous tous ses angles présente une histoire de la Famine qui repose sur une connaissance solide des principales questions. D'ailleurs, Kelly se montre plus malin qu'ó Murchadha en ce qui concerne le "Gros mot", même s'il eût été préférable, pour la première référence, de donner le titre complet de The Last Conquest of Ireland (Perhaps), car ce « perhaps » là avait son importance pour l'auteur : 
Dans The Last Conquest of Ireland, John Mitchel, l'un des pères fondateurs du nationalisme irlandais moderne, décrivit les notables britanniques qui étaient aux manettes pendant la famine comme des gargouilles génocidaires. Ce n'était pas le cas. Pour la plupart, c'étaient des hommes à l'esprit éveillé qui vivaient dans la crainte de Dieu et étaient (de leur propre avis) pleins de bonnes intentions (et c'est d'ailleurs cela qui les rend si déprimants). S'il y a une leçon à retenir de la famine, c'est celle du mal que sont capables de faire même les meilleurs d'entre les hommes lorsqu'ils sont perdus et laissent la religion et l'idéologie politique prendre le pas sur la raison et l'humanité.

Tout cela est "malin", mais plus loin : "I met Murder on the way / He had a mask like Castlereagh $\aleph^{72}$. Les politiciens et les bureaucrates sont bien responsables de leurs politiques, Ó Murchadha dit lui les choses telles qu'elles sont.

On trouve quelques erreurs typographiques (Peter Gray devient Peter Grey à la page 331, et p. 189 le quaker anglais William Forster devient William Foster quand son nom apparait pour la seconde fois dans le même paragraphe), mais ces erreurs sont peu nombreuses et pourront être corrigées dans une prochaine édition (et l'on ne doute pas qu'il y en ait plusieurs à venir). Il y a également quelques erreurs mineures qu'un historien formé en Irlande n'aurait sans doute pas faites et quelques formules qui peuvent paraître étranges à un lecteur irlandais. Par exemple, Mitchel est un enfant du presbytère (son père était pasteur presbytérien), pas un "enfant de l'Ascendancy ${ }^{73}$ " (p. 309), et la vente des droits locatifs ${ }^{74}$ était le plus souvent appelée «Ulster Custom » ou "Tenant Right » (pas «Ulster Right» [p. 303], même si ce terme a parfois servi), et d'autres choses de ce genre. Mais c'est pinailler de manière pédante que d'insister là-dessus. C'est un bon livre, qui fait ce que dit Bill clinton : il fournit « un récit édifiant » à propos des dirigeants bornés par leur idéologie politique, c'est-à-dire des gens comme Mitt Romney, Paul Ryan, Eric Cantor et les dirigeants républicains à la Chambre des représentants. Et c'est sans aucun doute, en partie, ce pour quoi il a été écrit.

On verra souvent The Graves are Walking de Kelly dans le métro new-yorkais, la ligne EL de Philadelphie, la L de Chicago ou la T de Boston, tandis que de nombreux exemplaires d' Ireland's Agony de Ó Murchadha seront vus dans les bus Feda O'Donnell, l'Enterprise qui va de Dublin à Belfast, et dans le métro londonien. Mais où donc verra-t-on The Curse of Reason de Delaney? C'est un livre très différent des deux autres. Il ne s'embarrasse pas de raconter « toute l'histoire » du début jusqu'à la fin. Il se concentre au contraire sur la vie et les écrits de quatre personnes: Trevelyan, Mitchel, MacHale et Elizabeth Smith, l'épouse d'un propriétaire terrien, qui tenait un journal. Une telle approche peut être confuse pour le lecteur qui voudrait simplement qu'on lui raconte les choses comme si c'était un "conte tout bête, sans fard ». Mais Delaney s'en sort bien. On aimerait qu'il mette au cœur de son récit la vie d'un membre des classes inférieures, comme Hugh Dorian par exemple, ou bien qu'il se serve de l'Irish Folklore Commission pour ajouter le point de vue de ces classes inférieures. Et il faut préciser que, d'après ma lecture, Delaney ne commet pas d'entorse aux formes traditionnelles (ni ne mélange les genres) comme Colm Tóibín semblait suggérer que les sources et le thème étudiés l'exigeraient. D'ailleurs, d'après moi, les prochains historiens de la Famine qui chercheraient à produire une enquête globale sur la catastrophe devraient tenter de développer de nouvelles manières de la raconter. Quoiqu'il en soit, l'approche de Delaney est innovante, et en choisissant de prendre une route qui n'avait encore jamais été empruntée, il ne méprise pas le lecteur qui connaît déjà l'essentiel de la question. Où trouvera-t-on le livre de 
Delaney? Dans les salons irlandais, britanniques et américains, entre les mains de gens qui savent apprécier l'histoire de première qualité.

Delaney, Kelly et ó Murchadha ont tous les trois produit des livres impressionnants. Ils feront tous leur chemin jusque dans les programmes universitaires, et tous les trois trouveront un lectorat plus large, car ils sont lucides, intelligents et touchants, chacun des trois ouvrages étant le fruit du travail d'un historien accompli (même si ce sont des historiens très différents) qui fait honneur à son éditeur. Mais à en juger par les étagères des librairies irlandaises et américaines (de moins en moins nombreuses), l'ouvrage qui aura le plus de lecteurs est le Famine Plot de Coogan, mal conçu et malhabilement écrit. Malheureusement, il ne s'agit ni d'un des meilleurs travaux sur la Famine, ni même d'un des meilleurs livres de Coogan. Et pourtant, à la lecture de la retranscription de son débat radiophonique avec Kennedy, il était difficile de ne pas me souvenir de ce petit garçon installé avec son père en 1974 pour regarder Mohamed Ali face à George Foreman, qui frappait plus fort - «the Rumble in the Jungle » ${ }^{75}$. Ce petit garçon fut horrifié chaque fois que son grand héros finissait contre les cordes, puis époustouflé lorsqu'il explosa dans le huitième round et mit son adversaire à terre. Coogan n'est pas un Mohamed Ali, et son ouvrage ne contient pas de huitième round, mais en poussant un éminent négationniste du génocide à juger la Grande Famine selon la Convention des Nations Unies, l'auteur et le journaliste a acculé un adversaire formidable et mis en évidence quelques-unes des faiblesses du révisionnisme de la fin du vingtième siècle ${ }^{76}$.

\section{« Vivez-vous loin d'ici, Votre Grâce ? »}

Felix Mendelssohn est mort à Berlin le 4 novembre 1847. Dans l'Ouest de l'Ulster, sa mort n'a pas beaucoup compté, si ce n'est pour un jeune agent des douanes de 23 ans qui travaillait dans une petite chambre du premier étage de l'hôtel Dillon, sur la place publique de Donegal Town. William Allingham, qui se faisait alors connaître en tant que poète, nota la mort du compositeur dans son journal le 13 novembre, alors qu'il travaillait sur son poème " The Ruined Chapel », qui paraîtrait dans le premier numéro de Harper's, le magazine littéraire new-yorkais, en 1850. Un peu plus de deux semaines plus tard, le dernier jour du mois, Allingham visita un hospice. Il n'en précise pas le nom dans son journal, mais ça devait être celui de Donegal ou celui de Ballyshannon. Tous deux étaient alors emplis de gens malades et abandonnés, sans terre et malchanceux, pour qui effectuer des corvées dans ces espaces confinés et infestés où les hommes et les femmes étaient séparés était ce que Hugh Dorian nomma «le dernière partie de toutes ». Là-bas, Allingham entendit une musique qui n'avait rien à voir avec celle de Medelssohn :

Le 30 novembre. Visite d'un hospice. Tom Read, un fou aux petits yeux noirs perçants : il se met parfois un morceau de fer sur la tête pour faire du bien à son cerveau. Joue du violon, il n'a plus que les deux premières cordes: "Ain kind Dearie ", "Pandun [sic] O'Rafferty ", en grognant et en grommelant sans arrêt, grommelant plus encore à chaque fausse note. Je lui promets des cordes. «Vivezvous loin d'ici, Votre Grâce ? " ${ }^{77}$

Le douanier n'indique pas sa réponse, et pour ce qu'on en sait la question de Tom Read était peut être amèrement rhétorique. Mais l'État enregistrait méticuleusement tout ce qui l'intéressait. Le lieu de résidence, le sexe, l'âge, la religion, le métier, l'état de santé et les dates d'arrivée et de départ de Read, toutes ces informations auront été enregistrées dans le registre de l'hospice. Et tout mauvais comportement de sa part y aura également été consigné. Malheureusement, les registres des deux hospices pour l'année 1847 ont été 
perdus ou détruits, et avec eux les traces laissées par cette vie $\mathrm{e}^{78}$. Ce nom de famille, qui n'est pas courant dans le sud du Donegal, fait penser que Read venait probablement de Pettigo, et se trouvait donc sans doute à l'hospice de Donegal. Et s'il venait effectivement de Pettigo, alors il était probablement protestant, car les Read/Reid de Pettigo étaient semble-t-il à l'époque soit des anglicans, soit des presbytériens, soit des méthodistes. Mais l'âge de Read, son état de santé, les dates auxquelles il est arrivé à l'hospice et en est reparti, ainsi que les conditions de son départ; tout cela ne peut désormais que faire l'objet de conjectures. Voici toutefois ce que l'on peut affirmer avec certitude: en novembre 1847, au moment où Read grattait ses cordes avec son archer, les hospices de Donegal et de Ballyshannon étaient tous les deux presque pleins et certains de leurs " pensionnaires » mourraient de la fièvre. Que Read soit mort ou ait survécu à l'hospice (et que le douanier soit ou non revenu avec les cordes), l'avenir que ses « compagnons d'infortune » et lui-même s'étaient imaginé leur était retiré par un champignon qui s'était d'abord attaqué à la pomme terre en 1845 et en 1846, et par la réponse apportée à ce champignon par le gouvernement du Royaume-Uni.

Les artistes de tous les styles (les équivalents actuels d'Allingham ou de Read, ainsi que les artistes visuels) devraient être tout aussi impliqués que les universitaires dans les commémorations publiques, qu'elles soient grandioses ou grotesques, de l'histoire irlandaise. Il est certain que pour les originaires du Donegal, qu'ils soient sur place ou à Glasgow, Londres, Philadelphie, New York, Chicago et au-delà, The Quiet Glen/An Gleann Ciúin du violoniste Tommy Peoples (une compilation de morceaux nouveaux et anciens, dont certains comme The Coffin Ships évoquent les problèmes de la Famine) a empêché le temps de faire disparaître les fantômes des années 1840, d'une manière qui n'est possible que pour les historiens pourvus du «don de ranimer la flamme de l'espoir dans le passé »79. Peoples est issu de la même tradition musicale que celui qui jouait la jigue Páidín ó Raifeartaigh alors que l'hospice se remplissait autour de lui. Son art n'est donc pas un simple hommage qui ne poserait pas de questions. Il protège Mary Gallagher ainsi que Hugh et Sarah Gallagher, qui lui ont coupé l'oreille à la faucille, de ceux qui préféreraient les laisser reposer en paix et passer à autre chose.

\section{NOTES}

2. Primo LEVI, Les Naufragés et les Rescapés : Quarante ans après Auschwitz [1986], traduit de l'italien par André Maugé, Paris : Gallimard, 1989, pp. 20 et 36-69 (« La Zone Grise »), p. 38 en particulier. 3. Peu d'ouvrages sur la Grande Famine utilisent les écrits de Primo Levi. Dans The Feminization of the Famine: Expressions of the Inexpressible (Cork: Cork University Press, 1997, p. 3), Margaret KELLEHER attribue ce fait à la conscience qu'ont les historiens des « dangers » des « analogies entre la Shoah et la Famine irlandaise des années 1840 » qui «risquent de devenir banales et simplistes, et d'estomper les complexités de chacune de ces catastrophes». Il y a quelques exceptions notoires, comme les travaux de Margaret Kelleher elle-même, ou l'article de Luke GIBBONS, « Words Upon the Windowpane : Image, Text, and Irish Culture », pp. 43-56 in James ELKINS (ed.), Visual Cultures , Chicago : University of Chicago Press, 2010. S'il ne fait pas référence spécifiquement à Primo Levi, Joe LEE travaille sur « ce que nous savons d'autres expériences humaines terribles, dont la Shoah 
n'est pas des moindres ", lorsqu'il réfléchit à l'expérience complexe de la Famine, dans une conférence animée, «Famine as History », pp. 159-175 in Cormac Ó GRÁDA (ed.), Famine 150: Commemorative Lecture Series, Dublin : Teagasc and UCD (voir en particulier les pages 167-169 et 172).

4. Note de traduction: intraduisible en français, le terme anglais soupers désigne les personnes qui acceptaient de se convertir au protestantisme en échange de "soupe » (c'est-à-dire de nourriture, mais aussi d'aide de manière plus générale) de la part d'évangélisateurs protestants.

5. $\mathrm{NdT}$ : intraduisible également, le terme grabbers désigne des paysans locataires qui récupéraient la terre d'autres paysans dans des conditions douteuses ou controversées.

6. Pour documenter la violence de cette difficulté à juger les affamés, le docteur Daniel O'Donovan, de Skibbereen dans le comté de Cork, mentionne l'histoire d'un garçon de 14 ans qui égorgea deux jeunes gens pour leur prendre une petite portion de bouillie, ou celles de mères arrachant la nourriture des mains de leurs propres enfants affamés, ou de pères et fils se bagarrant pour des pommes de terre. Voir Laurence M. GEARY, « What People Died of during the Famine ", in Ó GRÁDA, Famine 150, op. cit., pp. 95-112. À propos du cannibalisme, voir Cormac Ó GRÁDA, «Eating People is Wrong: Famine's Darkest Secret ?» (<http://papers.ssrn.com/sol3/ papers.cfm?abstract_id=2209806>, mis en ligne le 31 janvier 2013).

7. Hugh DORIAN, The Outer Edge of Ulster: A Memoir of Social Life in Nineteenth-Century Donegal, édition scientifique accompagnée d'une introduction de Breandán Mac Suibhne et David Dickson (Dublin : Lilliput Press, 2000 / Notre-Dame: University of Notre-Dame Press, 2001). L'édition américaine contient en plus une courte préface (pp. ix-xii) qui porte principalement sur l'histoire de la Famine. Par ailleurs, la pagination est inchangée. Un passage de cette préface (p. xi) est retravaillé dans le présent article. En plus du chapitre « The Years of the Famine » des mémoires de Dorian, on peut considérer qu'un autre chapitre, intitulé "The Landlord Extermination ", porte également sur la Famine, ainsi que d'autres passages du livre. On peut se laisser guider par les entrées du terme Famine listées dans l'index, mais il faut lire attentivement l'ensemble des mémoires de Hugh Dorian pour véritablement identifier toutes les références à la Famine.

8. Ici, on peut considérer que «l'entourage élargi » de Dorian se compose des circonscriptions électorales de Fanad West et Fanad North. D'après le recensement de 1851, la population respective de ces deux circonscriptions représentait $79,3 \%$ et $72,3 \%$ de ce qu'elle était en 1841 , soit cinq ans avant le pic de peuplement.

9. Ibid, p. 256.

10. Ibid, p. 130.

11. Ibid., pp. 216-217 (souligné par l'auteur). À propos du travail que les membres des comités d'aide aux victimes demandaient aux affamés, Dorian fait la remarque suivante : «cette manière détournée de rendre service ressemblait davantage à du travail forcé ou à une punition réservée aux condamnés".

12. Ibid., p. 223.

13. Ibid., pp. 41-43.

14. Ibid., pp. 172-173.

15. Ibid., p. 223.

16. Ibid., p. 230.

17. Ibid., pp. 214-215.

18. $\mathrm{NdT}$ : en français et en italique dans le texte.

19. $\mathrm{NdT}$ : voir note 5 .

20. Outer Edge of Ulster, op. cit., pp. 191-192. Voir aussi pp. 228-229, 239-240 et seq.

21. Ibid., p. 239.

22. Ibid., p. 191 et seq.

23. Ibid., pp. 227-229.

24. $\mathrm{NdT}$ : terme irlandais désignant l'histoire orale, la tradition orale, le folklore. 
25. $\mathrm{NdT}$ : « résidu » en irlandais.

26. Cathal PÓIRTÉIR (ed.), Glórtha ón Ghorta: Béaloideas na Gaeilge agus an Ghorta Mór, Baile Átha Cliath (Dublin, NdT) : Coiscéim, 1996, p. 69. Malheureusement, cette source de grande valeur ne précise pas les dates auxquelles les témoignages ont été enregistrés.

27. $\mathrm{NdT}$ : l'auteur précise que sa version anglaise est loin de rendre toute la violence de l'original en irlandais.

28. Voir note 20.

29. Cormac Ó GRÁDA, The Great Irish Famine, Houndmills : Macmillan, 1989, p. 77. Il se montre plus dur par ailleurs en décrivant le livre comme "une approche aseptisée et sans passion de la Grande Famine, qui domine désormais chez les historiens de l'Irlande». Colm TóIBÍN, dans "The Irish Famine » [1999], in Colm TÓIBÍN et Diarmaid FERRITER, The Irish Famine : a Documentary, Londres : Profile Books, 2001, pp. 3-4, 10-12, propose une analyse nettement plus longue et négative du style et de la méthode de ce «livre utile, qui avance à vive allure, mais s'attarde sur des détails et un examen exagérément attentif."

30. Voir en plus des compilations de Póirtéir l'ouvrage de Cormac Ó GRÁDA, An Drochshaol: Béaloideas agus Amhráin, Baile Átha Cliath (Dublin, NdT) : Coiscéim, 1994, surtout pp. 17-19 et 24-26. Version en anglais: Black '47 and Beyond: the Great Irish Famine in History, Economy and Memory, Princeton : Princeton University Press, 2000, pp. 194-225.

31. $\mathrm{NdT}$ : dérivé du terme souper, expliqué à la note 4 .

32. $\mathrm{NdT}$ : voir note 5 .

33. $\mathrm{NdT}$ : voir note 20 .

34. Gearóid Ó TUATHAIGH, Ireland Before the Famine [1972], Dublin : Gill and Macmillan, 2007, p. 184.

35. $\mathrm{NdT}$ : voir note 5.

36. TÓIBÍN, « Irish Famine », op. cit., pp. 22-24. Tóibín l’a écrit avant la publication de l'ouvrage référence d'Angela BOURKE, The Burning of Bridget Cleary : A True Story (New York : Penguin, 1999), l'un des livres les plus innovants en matière d'histoire sociale et culturelle en Irlande, dans laquelle l'histoire orale occupe une place centrale.

37. TÓIBÍN, « Irish Famine », op. cit., pp. 28-29

38. $\mathrm{NdT}$ : l'auteur fait référence aux grèves de l'hygiène et de la faim menées par des prisonniers membres de l'IRA pour obtenir le statut de prisonniers politiques de la part du gouvernement britannique dans les années 1980. Ils étaient détenus dans les prisons de Long Kesh et d'Armagh, en Irlande du Nord.

39. NdT : titre d'une chanson de Johnny Cash sur l'Irlande (Forty Shades of Green, 1959) ; le vert, traditionnellement associé à l'île d'émeraude, est également symbole du nationalisme irlandais.

40. $\mathrm{NdT}$ : expression de l'auteur qui fait référence au nationaliste des années 1840, John Mitchel. Membre de la Jeune Irlande, groupe qui prônait un nationalisme culturel et constitutionnel, les horreurs de la Famine le poussèrent à se radicaliser et à évoluer vers le socialisme agraire révolutionnaire. Après la révolte de 1848, il fut condamné à un exil de 14 ans et envoyé aux Bermudes, puis en Tasmanie, d'où il s'échappa pour les États-Unis.

41. Ó GRÁDA, Great Irish Famine, op. cit., p. 11.

42. Parmi ses nombreux ouvrages sur le sujet, on peut lire Genocide in International Law: The Crime of Crimes, Cambridge : Cambridge University Press, 2000.

43. Pour ne pas accabler ó Murchadha, il faut reconnaître que la sentence de Mitchel fait souvent l'objet de citations erronées, dans lesquelles le verbe «créer» est remplacé par «faire» ou « envoyer".

44. $\mathrm{NdT}$ : « the G-word » en anglais.

45. L'expression apparaît de façon récurrente dans l'étude de la politique étrangère des ÉtatsUnis menée par Samantha POWER (et qui lui a valu le prix Pulitzer) : ‘A Problem from Hell': America and the Age of Genocide, New York : Harper, 2003. 
46. Sur la question de l'intention et de la législation sur le génocide, qui préoccupe tant Kennedy, voir SCHABAS, Genocide in International Law, op. cit., p. 213 et seq., en particulier les pages 222-226 sur les preuves d'intention.

47. Carlo GINZBURG, Le Juge et l'Historien : Considérations en marge du procès Sofri [1991], traduction collective de l'italien, édition brochée épuisée, Lagrasse : Éditions Verdier, 1998, p. 15.

48. Cité in ibid., p. 15

49. Peter NOVICK, That Noble Dream: The 'Objectivity Question' and the American Historical Profession, Cambridge : Cambridge University Press, 1988.

50. Seamus DEANE, « Wherever Green is Read », pp. 91-105 in Máirín NÍ DHONNCHADHA et Theo HORGAN (eds), Revising the Rising, Derry : Field Day, 1991. Il a été republié dans Ciarán BRADY (ed.), Interpreting Irish History: The Debate on Historical Revisionism, Dublin : Irish Academic Press, 1994, pp. 234-45.

51. LEE, "The Famine as History ", op. cit., p. 166, fait référence à ce travail des méninges. D'après lui, depuis 1969, « les universitaires qui craignaient que leur recherche ne fournisse des arguments à l'IRA pour son interprétation de l'histoire irlandaise » ont eu "tendance à minimiser l'échelle de la Famine ainsi que la responsabilité des politiciens britanniques ».

52. $\underline{\mathrm{NT}}$ : référence à Yeats, qui a produit de nombreux écrits (poèmes et pièces de théâtre) exaltant le nationalisme et se demandait à la suite du soulèvement de Pâques 1916 s'il en portait une part de responsabilité. Il a écrit dans un poème : «Did that play of mine send out / Certain men the English shot? ", à propos de sa pièce Cathleen Ní Houlihan.

53. Liam KENNEDY, Two Ulsters : A Case for Repartition, Belfast : Queen's University, 1986.

54. Peter GRAY, «Ideology and the Famine », in PÓIRTÉIR, Great Irish Famine, op. cit., pp. 86-103, 102-103 (souligné par l'auteur). David P. NALLY, Human Encumbrances: Political Violence and the Great Irish Famine, Notre Dame : University of Notre Dame Press, 2011, p. 226, dit à propos de ce passage : «Il s'agit là d'une des dénonciations les plus fortes de ces dernières années. Toutefois, puisque Gray introduit ici le vocabulaire de la responsabilité ("négligence coupable") tout en repoussant l'accusation de génocide, il faut se demander dans quel sens il utilise le terme coupable. "

55. SCHABAS, Genocide in International Law, op. cit., p. 1 (souligné par l'auteur).

56. Voir par exemple, James S. DONNELLY Jr., " Mass evictions and the Great Famine », pp. 155-73 in PÓIRTÉIR, Great Irish Famine, op. cit., et «Constructing the Memory of the Irish Famine, 1850-1900 ", Éire-Ireland, Vol. 31, No. 1-2, 1996, pp. 26-61. Les deux articles ont été à nouveau publiés dans son ouvrage Great Irish Potato Famine, op. cit., pp. 209-45.

57. Ballyshannon Herald, 29 janvier 1847.

58. Ibid., 8 janvier 1847.

59. Ibid., 15 janvier 1847.

60. Ibid., 29 janvier 1847.

61. Ibid., 12 février 1847.

62. National Archive of Ireland, Outrage Papers 7/113 Donegal, 6 mai 1848, Thomas E. Blake, RM, à Redington.

63. $\mathrm{NdT}$ : ici, l'auteur fait référence à Cromwell, qui expulsa les propriétaires catholiques de leurs terres pour les remplacer par des anglicans. Les récalcitrants étaient massacrés, d'où l'expression qui veut qu'ils étaient envoyés «vers le Connaught ou vers l'enfer » (« to Connaught or Hell » en anglais), le Connaught étant la province qui se trouve le plus à l'Ouest de l'Irlande, la plus pauvre, et celle dont les terres sont les plus difficile à exploiter. Par conséquent, elle était d'un intérêt moindre aux yeux des colons britanniques, qui suggéraient aux Irlandais expulsés de leurs terres de s'y rendre.

64. $\mathrm{NdT}$ : il s'agit d'une erreur typographique sur le nom d'un des deux principaux partis politique d'Irlande: le Fianna Fáil, l'autre parti étant le Fine Gael. L'erreur est d'autant plus gênante que l'Irish Press était l'organe du Fianna Fáil.

65. $\mathrm{NdT}$ : nom d'un régiment de l'armée britannique. 
66. Ruán O’DONNELL, The Rebellion in Wicklow, 1798, Dublin : Irish Academic Press, 1998, p. 144.

67. $\mathrm{NdT}$ : l'erreur typographique n'étant pas traduisible, le choix a été fait de conserver la citation en anglais. La version de Coogan voudrait dire qu'on coupait les cuisses et les hanches des femmes au sens où on les leur enlevait, tandis que la version de Ruán O'Donnell signifie qu'on leur faisait des coupures sur les cuisses et les hanches.

68. DONNELLY, Great Irish Potato Famine, op. cit., p.245.

69. $\mathrm{NdT}$ : nom officiel du mouvement religieux Quaker.

70. Référence à la révolte avortée organisée en août 1848 par des membres de la Jeune Irlande.

71. Voir par exemple, de Christine KINEALY, « Potatoes, Providence and Philanthropy: the role of Private Philanthropy during the Irish Famine», pp. 140-71 in Patrick O'SULLIVAN (ed.), The Meaning of the Famine, London and New York : Leicester University Press, 1997.

72. $\mathrm{NdT}$ : citation du poète Percy Shelley qui fait référence à Castlereagh, homme d'État affable connu pour avoir justifié le «massacre de Peterloo ", résultat d'une manifestation de radicaux et de réformateurs à Manchester en 1819, ce qui lui a valu de devenir un symbole du conservatisme réactionnaire.

73. $\mathrm{NdT}$ : sans équivalent français, le terme Ascendancy désigne en Irlande les membres anglicans des classes dirigeantes.

74. $\mathrm{NdT}$ : le Tenant Right ou Ulster Custom désigne un système développé en Ulster selon lequel les paysans avaient la possibilité de transmettre la terre qu'ils louaient en obtenant une compensation financière pour les améliorations apportées à leur parcelle pendant la durée de leur location. La généralisation de cette coutume à l'ensemble de l'Irlande fut l'une des revendications principales de l'agitation nationaliste agraire de la deuxième moitié du dixneuvième siècle.

75. $\mathrm{NdT}$ : « le grondement dans la jungle », surnom donné au combat de boxe organisé à Kinshasa entre le champion du monde poids lourd en titre, Foreman, et son challenger, Ali, qui remporta la victoire par $\mathrm{KO}$ au $8^{\mathrm{e}}$ round.

76. Je ne suis probablement pas le seul à penser cela. Voir par exemple le commentaire par Mick HEANEY (paru dans l'Irish Times du 5 janvier 2013) d'une autre émission de radio à laquelle participait Coogan, l'émission Blighted Nation de Myles Dungan, : « [Si] sa rhétorique moralisatrice et emprunte de nationalisme manque de subtilité, l'insistance de Coogan sur les souffrances des victimes de la Famine en appelait davantage à la sympathie de l'auditeur que l'approche scrupuleuse et équitable de [Mary] Daly et [Peter] Gray, qui accordent aux instances dirigeantes une approche peut être plus équilibrée que nécessaire. Certes l'objectivité peut être fondamentale pour la recherche universitaire, mais dans ce contexte d'une émission d'histoire populaire, le ton polémique de Coogan a plus de résonance. "

77. William ALLINGHAM, A Diary, édité par H. ALLINGHAM et D. RADFORD, Londres : Macmillan, 1907, p. 41.

78. Tous les manuscrits portant sur le fonctionnement de l'hospice de Donegal (Donegal Union Workhouse) ont été détruits dans un incendie. Les livres des minutes de l'hospice de Ballyshannon (Ballyshannon Union) ont été conservés pour cette période, mais pas le registre. Je remercie Niamh Brennan, archiviste du conté de Donegal, pour nos discussions au sujet de ces dossiers, qu'elle m'a communiqués le 26 mars 2013.

79. Walter BENJAMIN, "Theses on the Philosophy of History», in Illuminations: Essays and Reflections [1968], édité par Hannah Arendt, traduit de l'allemand vers l'anglais par Harry Zohn, New York : Shocken, 2007, p. 255. 


\section{RÉSUMÉS}

La notion de la «zone grise », définie par Primo Levi, permet de mieux comprendre la vie des pauvres pendant la Grande Famine. Les mémoires de Hugh Dorian et les archives de l'Irish Folklore Commission mettent en lumière les tensions entre Irlandais à cette période, ainsi que leur attitude vis-à-vis du gouvernement. La question de la responsabilité de Londres apparaît également dans les ouvrages récents et innovants de Tim Pat Coogan, Enda Delaney, John Kelly et Ciarán ó Murchadha, même si chacun apporte une réponse différente à la question éminemment sensible d'un génocide.

The notion of a 'grey zone' defined by Primo Levi helps to better understand the lives of the poor during the Great Famine. Hugh Dorian's memoirs and the archives of the Irish Folklore Commission highlight tensions between Irish people at the time, as well as their attitude towards the government. The issue of London's responsibility is also dealt with by recent innovative books by Tim Pat Coogan, Enda Delaney, John Kelly and Ciarán Ó Murchadha, even if each one provides a different answer to the highly-charged question of a genocide.

\section{AUTEURS}

BREANDÁN MAC SUIBHNE

Centenary College, New Jersey 